\title{
Fast lysis of Escherichia coli filament cells requires differentiation of potential division sites
}

\author{
Miguel Angel de Pedro, ${ }^{1}$ Joachim-Volker Höltje ${ }^{2}$ and Heinz Schwarz ${ }^{2}$
}

Author for correspondence: Miguel Angel de Pedro. Tel: +34 91 3978083. Fax: + 34913978087.

e-mail:madepedro@cbm.uam.es

1 Centro de Biología Molecular 'Severo Ochoa' Consejo Superior de Investigaciones CientíficasUniversidad Autónoma de Madrid, Campus de Cantoblanco, 28049 Madrid, Spain

2 Max-Planck-Institut für Entwitcklungsbiologie, Spemannstrasse 35, D-72076 Tübingen, Germany

\begin{abstract}
Periodic activation of zonal peptidoglycan (murein) synthesis at division sites in Escherichia coli has been reported recently. Zonal synthesis is responsible for septum formation, whereas elongation of the cell sacculus is performed by diffuse insertion of precursors. Zonal synthesis can be triggered in ftsA, fts $Q$ and ftsl (pbpB) division mutants growing as filaments at the restrictive temperature, but not in fts $Z$ mutant strains. The lytic response to $\beta$-lactams of cells able or unable to periodically trigger a zonal mode of murein synthesis could be substantially different. Therefore, we investigated the response to the bacteriolytic $\beta$-lactam cefsulodin of $\mathrm{fts} Z$ and $\mathrm{ftsl}$ mutants growing at the restrictive $\left(42{ }^{\circ} \mathrm{C}\right)$ temperature. The $\mathrm{ftsl}$ cells lysed early and quickly after addition of the antibiotic. Sacculi of lysed cells were transversely cut in a very sharp way. In contrast the fts $Z$ strain lysed late and slowly after addition of the antibiotic and sacculi showed a generalized weakening of the murein network and extended breaks with a frayed appearance. No transversely cut sacculi comparable to those seen in the ftsl samples were found. Our results strongly support that $\beta$-lactam-induced lysis occurs preferentially at division sites because of the activation of zonal murein synthesis at the initiation of septation.
\end{abstract}

Keywords: $f t s Z, p b p B$, penicillin-binding protein, beta-lactam, murein

\section{INTRODUCTION}

The peptidoglycan (murein) layer of the bacterial cell envelope forms a giant, bag-shaped macromolecule, the sacculus, which surrounds the cell. The sacculus is the principal stress-bearing element of the cell wall and plays an essential role in bacterial morphogenesis (Ayala et al., 1994; Höltje, 1998; Nanninga, 1998). Metabolism of the sacculus requires a large number of enzymes and metabolites and is subjected to complex regulatory pathways (Glauner \& Höltje, 1990; Höltje, 1998; Höltje \& Glauner, 1990; Nanninga, 1991). The physical integrity of the sacculus is of vital importance to the cell because it is the only structure able to withstand the turgor pressure of the cell. Therefore, growth and septation of the sacculus must proceed avoiding the generation of discontinuities to prevent cell lysis (Koch, 2000).

Abbreviations: PBP, Penicillin-binding protein; SMS, septal murein synthesis.
In Escherichia coli the mode of insertion of new precursors into the sacculus apparently shifts from diffuse to zonal at the initiation of septation (de Pedro et al., 1997). Lateral wall elongation is accomplished by intercalation of new and pre-existing murein all over the cylindrical surface of the sacculus. At the time of septation an area of highly localized murein synthesis develops into the potential division site and remains active until septum completion (SMS, septal murein synthesis). Once the dividing sacculus is split, SMS stops and the new cell wall poles become metabolically inert. Activation of SMS requires the activity of FtsZ, but not the essential division proteins FtsA, FtsQ and PBP3 (penicillin-binding protein 3). Inactivation of FtsZ leads to the generation of filamentous cells in which murein precursors are inserted in a diffuse way for as long as the filament elongates. In contrast, cells from $f t s A, f t s Q$ or ftsI mutants growing at the restrictive temperature are able to trigger SMS in due time. However, SMS remains active only for a defined period of time. Then it is switched off and murein synthesis reverts to a diffuse insertion mode. When the time for the next round of 


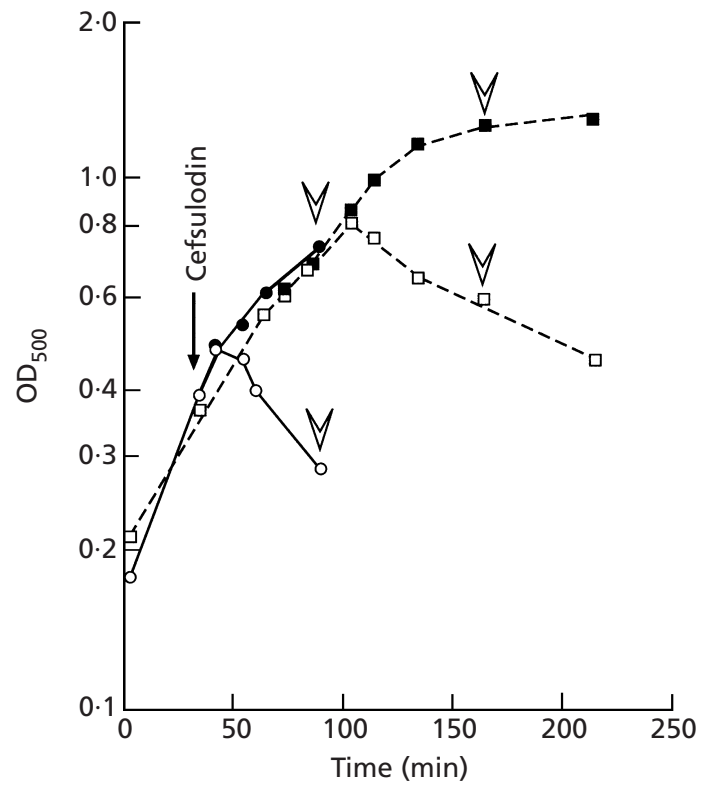

Fig. 1. Effect of cefsulodin on filament cells of E. coli MC6RP41 (ftsl) and MC6RP62 (ftsZ) growing at $42{ }^{\circ} \mathrm{C}$. Cultures of both strains growing exponentially at $30^{\circ} \mathrm{C}$ for three mass doublings were diluted $1: 4$ in the same medium prewarmed at $42^{\circ} \mathrm{C}$ (time 0 ) and allowed to grow for one more doubling in mass. At that moment (arrow) cultures were divided into two equal parts. Cefsulodin $\left(50 \mu \mathrm{g} \mathrm{ml}^{-1}\right)$ was added to one subculture of each strain and the $\mathrm{OD}_{550}$ of the cultures was measured periodically. At the times indicated by the arrowheads samples were removed from each subculture to purify sacculi for electron microscopy. O, MC6RP41 control; O, MC6RP41 plus cefsulodin; $\square$, MC6RP62 control; $\square$, MC6RP62 plus cefsulodin.

septation is reached and FtsZ assembles into new Zrings, SMS is triggered again and the whole process repeats. The consequences are that diffuse and zonal modes of murein synthesis alternate and that filaments of $f t s A, f t s Q$ or $f t s I$, but not $f t s Z$, mutants develop rings of 'all new' murein at the potential division sites (de Pedro et al., 1997).

Growth of the bacterial cell sacculus is thought to require the concerted action of biosynthetic and hydrolytic enzymes (Höltje, 1996, 1998; Höltje \& Heidrich, 2001; Weidel \& Pelzer, 1964). The former are essential enzymes which mediate elongation of nascent peptidoglycan strands as well as their insertion into the sacculus. An essential role for murein hydrolases has been repeatedly postulated on theoretical grounds. As the sacculus is a covalently closed structure, its enlargement requires cleavage of pre-existing covalent bonds (Höltje, 1998; Shockman \& Höltje, 1994; Weidel \& Pelzer, 1964). Nevertheless, none of the E. coli murein hydrolases known seems to be essential for enlargement. Even multiple mutants are capable of essentially normal growth in length (Höltje \& Heidrich, 2001; Shockman \& Höltje, 1994). In contrast, a critical requirement of murein hydrolases for cell division has been substantiated (Blackman et al., 1998; Heidrich et al. 2001; Höltje \& Heidrich, 2001; Tomasz, 1974). In E. coli the activity of amidases, acting in concert with endopeptidases and lytic translgycosylases, is necessary for proper septum splitting (Heidrich el al. 2001; Höltje \& Heidrich, 2001). This situation has led to the recent proposal of a model in which only septation would require the concerted action of synthetases and hydrolases. Enlargement of the sacculus would be mediated by murein transferases (Höltje \& Heidrich, 2001). It is speculated that enzymes exist that catalyse a transpeptidation between old and nascent murein by using the chemical energy present in pre-existing peptide crossbridges. Such enzymes would cleave bonds in the sacculus concomitantly with the insertion of new material, therefore obviating the need for additional hydrolases.

Induction of bacterial lysis by $\beta$-lactams and other murein synthesis inhibitors normally requires active growth of the cells and the activity of murein hydrolases (autolysins) (Tomasz, 1974; Tuomanen \& Tomasz, 1990; Leduc \& van Heijenoort, 1980; Leduc et al., 1982). These observations have led to models proposing that lysis occurs because the inhibitors break the balance between biosynthetic and hydrolytic enzymes, favouring uncontrolled murein degradation by the latter (Koch, 2000; Rogers et al., 1980; Weidel \& Pelzer, 1964). However, the lack of hard evidence in support of the implication of hydrolases in elongation of the sacculus and the demonstration of $\beta$-lactam-induced lysis in nongrowing cells under specific conditions (Ishiguro \& Kusser, 1988; Kusser \& Ishiguro, 1986, 1987) has led to alternative models in which lysis is the consequence of the activation of lytic enzymes which under normal growth conditions would be partially or totally inhibited (Ishiguro \& Kusser, 1988; Tomasz, 1983, 1984).

Early observations of $\beta$-lactam-induced bacteriolysis showed the preferential occurrence of lysis at potential division sites (Staugaard et al., 1976; Tomasz, 1974), which, in the case of E. coli, often resulted in sacculi with a sharp cut at the corresponding location (Schwarz et al., 1969). These observations suggest that SMS might be particularly prone to provoke lysis when disturbed. The zonal nature of SMS suggests that the local concentration of biosynthetic complexes might be higher at division sites than anywhere else in the cell. If biosynthetic complexes require the concomitant activity of hydrolases, damage inflicted by bacteriolytic agents should be more pronounced at the places with higher local densities. Furthermore, as septation itself needs the activity of murein hydrolases to split nascent septa (Heidrich et al. 2001; Höltje \& Heidrich, 2001), bacteriolytic agents could trigger lysis more effectively at those areas where hydrolases are actually performing their function and loss of control would have immediate consequences. According to such ideas, the mode of lysis of filament cells should be strongly dependent on whether or not SMS is activated during growth under restrictive conditions. In the former case lysis should occur as sharp cuts at the position of the rings of 'all new' murein generated by the activation of SMS. In the latter, as no regions of localized synthesis develop, a 
general weakening of the sacculus network, with essentially random break points, is more likely to be observed. Examination of cefsulodin-induced lysis in sacculi from filament cells of E. coli ftsZ and ftsI mutants confirmed these predictions.

\section{METHODS}

Strains and growth conditions. E. coli strains used in this work were MC6RP1 (K-12, $\mathrm{F}^{-}$dra drm leuA lys A proA thi thr) (Prats \& de Pedro, 1989), and derivatives MC6RP41 $\left(f t s I^{655}\right.$ ) and MC6RP62 ( $f t s Z^{84}$ ) (Garcia del Portillo \& de Pedro, 1990). Cultures were routinely grown in LB medium (Lennox, 1955) at 30 or $42{ }^{\circ} \mathrm{C}$, as indicated, in gyratory water baths. Growth was monitored by measuring $\mathrm{OD}_{550}$ in a Spectronic 20 (Bausch \& Lomb) spectrophotometer.

Purification of sacculi. Culture samples (ca $10^{10}$ cells) were harvested by centrifugation $(10000 \mathrm{~g}, 3 \mathrm{~min})$ at the growth temperature. The pellets were quickly resuspended into $3 \mathrm{ml}$ $0.9 \mathrm{~g} \mathrm{NaCl} \mathrm{l}^{-1}$, transferred into test tubes containing $6 \mathrm{ml} 8 \%$ $(\mathrm{w} / \mathrm{v})$ SDS and kept closed on a boiling water bath for $6 \mathrm{~h}$ with magnetic stirring. Sacculi were further processed for electron microscopy as described by de Pedro et al. (1997).

Electron microscopy of sacculi. Carbon-pioloform-coated copper grids (200\# mesh) were glow-discharged (10 min) and floated for $15 \mathrm{~min}$ on drops of sacculi suspensions appropriately diluted in double-distilled water. Grids were then removed, excess liquid was absorbed on filter paper and grids were allowed to air dry for $10 \mathrm{~min}$. Grids were washed (five times) by flotation on distilled water drops, stained by floating for $1 \mathrm{~min}$ on $1 \%$ (w/v) uranyl acetate in water, briefly washed in water and air-dried. To better visualize the flatness of sacculi, grids were in some instances subjected to carbon/ platinum shadowing at an angle of $15^{\circ}$. Microscopic observations were performed on a Philips CM10 transmission electron microscope at an acceleration voltage of $60 \mathrm{kV}$.

Muramidase digestion of murein sacculi. 'On the grid' digestion of sacculi was performed as described by de Pedro et al. (1997). In short, purified sacculi were deposited on electron microscope copper grids and washed as described above. Grids were floated for $1 \mathrm{~min}$ on drops $(50 \mu \mathrm{l})$ of a solution of Cellosyl muramidase (Aventis) at $5 \mu \mathrm{g} \mathrm{ml}^{-1}$ in $20 \mathrm{mM}$ sodium phosphate buffer, $\mathrm{pH} 4 \cdot 9$, at room temperature. To stop the reaction the enzyme solution was removed from the grid with filter paper, grids were rapidly washed three times in drops of precooled $\left(4-6{ }^{\circ} \mathrm{C}\right)$ double-distilled water and stained for $1 \mathrm{~min}$ by flotation on drops of $1 \%(\mathrm{w} / \mathrm{v})$ uranyl acetate in water.

\section{RESULTS}

\section{Cefsulodin-induced lysis in filament cells of $E$. coli MC6RP41 (ftsl) and MC6RP62 (ftsZ)}

Lysis was induced by addition of cefsulodin, a highly bacteriolytic $\beta$-lactam that binds preferentially to PBPs $1 \mathrm{a}$ and $1 \mathrm{~b}$ in E. coli and that has been used to study the mechanism of bacterial lysis (Garcia del Portillo et al., 1989; Jacoby \& Young, 1991; Powell \& Young, 1991). Cultures of both strains were grown in LB at $30^{\circ} \mathrm{C}$ for three mass doublings and were then diluted $1: 4$ into new medium pre-warmed at $42{ }^{\circ} \mathrm{C}$. Cultures were allowed to grow for one doubling in mass, divided into two halves and cefsulodin $\left(50 \mu \mathrm{g} \mathrm{ml}^{-1}\right)$ was added to one of the subcultures. Lysis started earlier and was clearly faster in the ftsI strain than in the fts $Z$ strain (Fig. 1).

\section{Analysis of sacculi from lysing cultures of $E$. coli MC6RP41 (ftsI) and MC6RP62 (ftsZ)}

At the times indicated by the arrowheads in Fig. 1, samples of filamenting cells were withdrawn from the cultures and processed for sacculi purification and electron microscopy. Sacculi from cefsulodin-treated ftsI cells showed evident differences compared to those from intact cells (Fig. 2a, b). Sacculi from treated cells were often transversely cut in an extremely sharp way (Fig. 2e, f). Rings of murein with a partially degraded appearance were often observed at positions apparently corresponding to division sites (Fig. 2b, e, f). The rest of the sacculus surface showed signs of general degradation in the form of a murein network mesh opening (Fig. 2c, d). In samples from similarly treated $f t s Z$ cultures only a few sacculi showed clear signs of degradation. Not a single one was found with cuts comparable to those in ftsI sacculi. Instead extended areas of partially degraded murein with a frayed appearance were found in sacculi from the fts $Z$ mutant (Fig. 3a). However, the murein of most sacculi also showed clear signs of general degradation, in the form of small openings and a fibrillar appearance (Fig. 3b, c), similar to that commented on above for ftsI strain filaments (Fig. 2c, d). The appearance of murein in antibiotic-treated $f t s Z$ sacculi was very similar to that of control sacculi subjected to mild digestion (1 $\mathrm{min}, 5 \mu \mathrm{g} \mathrm{ml} \mathrm{m}^{-1}$ at room temperature) with the murein hydrolytic enzyme Cellosyl, an endo- $N$ acetylmuramidase able to fully degrade murein into soluble muropeptides (Fig. 3c, d).

\section{Size distribution of sacculi from cefsulodin-lysed filamentous cells of E. coli MC6RP41}

An immediate consequence of the apparently preferential lysis at division sites in the ftsI strain is that the length distribution of lysed sacculi and the distribution of lengths from the cell poles to division sites should correspond. Therefore, the lengths of lysed sacculi from a culture of MC6RP41 grown at $42{ }^{\circ} \mathrm{C}$ for one doubling time and then treated with cefsulodin for 60 min were compared to the polar distances of constrictions in sacculi from filaments of a parallel antibiotic-free culture. For cefsulodin-treated sacculi, length was calculated from the rectangular coordinates of the sacculi ends using the microscope built-in system for grid position determination. To define the polar distance distribution of constrictions, the distance of each constriction to each pole was measured in the same way. Only straight sacculi were considered suitable. Repeated (10-fold) measurements of selected sacculi indicated a $\sigma$ of $\pm 0 \cdot 1 \mu \mathrm{m}$. The results (Fig. 4) showed a clear correlation between the two distributions, at least for sacculi up to $6 \mu \mathrm{m}$, and therefore support the idea that 

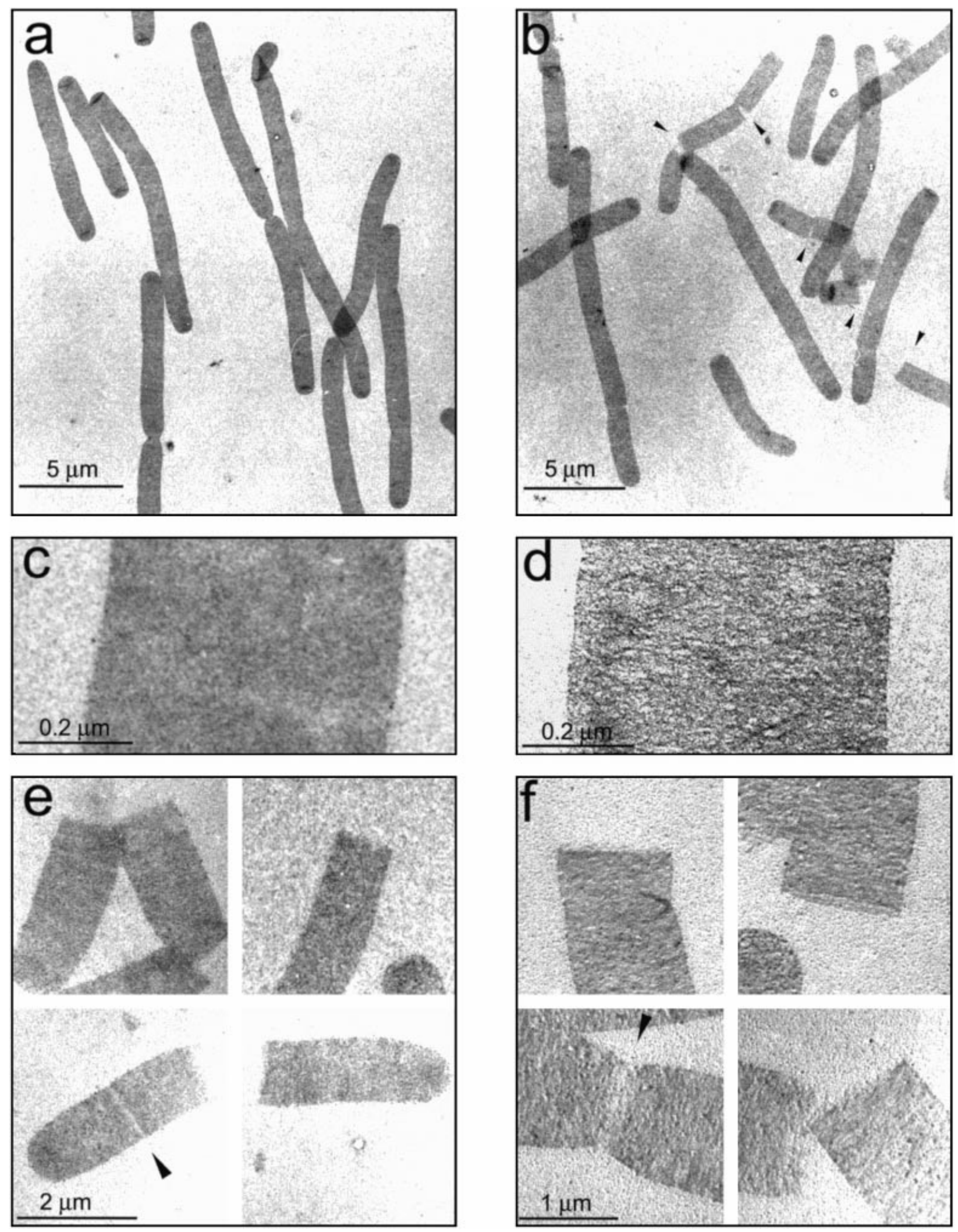

Fig. 2. For legend see facing page. 
cells do lyse at potential septation sites under our experimental conditions.

\section{DISCUSSION}

Analysis of sacculi from fts $Z$ and ftsI cell division mutants induced to lyse under restrictive conditions further confirmed that cell lysis is preferentially triggered at active growth sites (Höltje, 1995; Höltje \& Heidrich, 2001; Rogers et al., 1980; Schwarz et al., 1969; Tuomanen \& Tomasz, 1990; Tomasz, 1983; Weidel \& Pelzer, 1964) and suggests a co-localization of biosynthetic complexes and hydrolytic activities. Induction of cell lysis in filament cells of the fts $Z$ mutant by cefsulodin treatment resulted in rather delayed onset of lysis, as asserted by optical density measurements and in the generation of areas of extended damage apparently at random in the murein sacculus. On the assumption that hydrolases are associated to biosynthetic complexes (Höltje, 1996), such a pattern fits well with a diffuse mode of growth where many biosynthetic complexes are active at dispersed and random locations of the sacculus. Inhibition of each one would be insufficient to start lysis at that particular site, at least in the short term. However, accumulation of relatively small lesions would progressively reduce the mechanical resistance of the sacculus below the limit necessary to withstand the turgor pressure of the cell and lysis would ensue. In contrast, similarly treated $f t s I$ cells lysed faster and sacculi exhibited extremely sharp transverse cuts at positions which correspond with SMS activation sites in the ftsI cells. These characteristics suggest that the simultaneous inhibition of the synthetic enzymes in a relatively large number of densely packed biosynthetic complexes leads to rapid murein degradation at the precise place that breaks apart under the turgor pressure of the cell. In the $f t s I$ filaments, $\beta$-lactams also inhibit complexes involved in lateral wall synthesis. However, development of critical damage in these sites would be much slower than in regions of active SMS and cells would preferentially lyse at the latter sites as observed. An alternative possibility would be the activation of specific hydrolases at potential division sites in an FtsZ-dependent fashion. However, this alternative would not explain damage to murein outside the division site and so far there is no indication for direct regulation of murein hydrolases by FtsZ.

The periodic alternating use of diffuse and zonal modes of murein growth helps to explain the old observation that $\beta$-lactams apparently induce lysis in accordance with cell division in normally dividing cells. Induction of lysis by $\beta$-lactams proceeds at a rate related to the growth rate and very often cells blow up at positions corresponding to division sites (Leduc \& van Heijenoort, 1980; Leduc et al., 1982; Schwarz et al., 1969; Staugaard et al., 1976). According to our interpretation in any cell able to trigger SMS, lysis should occur preferentially at the division site. As generation of division sites is intimately related to growth rate, the rate of lysis should be automatically linked to the rate of growth.

The link between induction of fast, localized cell lysis and activation of SMS at division sites could explain previous, and apparently contradictory, results about the lytic response to cefsulodin of synchronized cultures of E. coli. In experiments performed with cells synchronized by thymine/amino acid starvation, addition of cefsulodin at any time prior to termination of DNA replication resulted in cell lysis at the time of division, but not earlier (Garcia del Portillo et al., 1989). In contrast, in similar experiments performed with cells synchronized either by the 'Baby Cell Machine' (membrane elution) (Jacoby \& Young, 1991) or centrifugal elutriation (Wientjes \& Nanninga 1991) methods, lysis started a short time (10 and $20 \mathrm{~min}$, respectively) after addition of the $\beta$-lactam with no apparent connection to the division event. The key fact might be that the thymine/amino acid starvation synchronization method blocks division via FtsZ, whereas both the Baby Cell Machine and centrifugal elutriation methods do not. Therefore, in the former case no cell in the culture was able to trigger SMS before the end of the ongoing round of DNA replication. Addition of cefsulodin during the replication period surely affected diffuse lateral wall synthesis. However, as shown above, cells take a long time to lyse when only lateral wall synthesis is affected. Therefore, cells could terminate replication, trigger SMS and, as cefsulodin was present, a fast lysis would then follow. In Baby Cell Machine and centrifugal elutriation synchronized cultures alignment of division initiation is not quite as strict as it is for thymine/amino acid starvation where the cells have to go through a complete round of replication (around $40 \mathrm{~min}$ under the conditions used) before SMS can actually start. Furthermore, the doubling time in the experiments was relatively short (30-60 min) and cefsulodin, as for any other $\beta$-lactam, requires a few minutes before the onset of lysis. Therefore, it is quite possible that SMS was triggered in some cells before or shortly after addition of cefsulodin to the synchronized cultures even at the earlier times of drug addition. Then lysis could start soon after cefsulodin addition in all instances, as was indeed observed. The longer lysis delay observed in centrifugal elutriation synchronized cultures could reflect the better synchrony apparently obtained by this method.

Fig. 2. Electron microscopy of sacculi from cefsulodin-treated filament cells of $E$. coli MC6RP41 (ftsl) growing at $42^{\circ} \mathrm{C}$. Samples from control and cefsulodin-treated cultures of MC6RP41 growing at $42{ }^{\circ} \mathrm{C}$ were collected as indicated in Fig. 1 and processed for electron microscopy. (a) Control sacculi; (b) cefsulodin-treated sacculi (arrowheads indicate break points); (c) detail of murein from control sacculi; (d) detail of murein from cefsulodin-treated sacculi; (e, f) lysed sacculi from cefsulodin-treated samples (arrowheads indicate partially degraded constriction sites). (a-e) Uranyl-acetate-stained sacculi; (f) uranyl-acetate-stained sacculi were shadow-cast with carbon-platinum at an angle of $15^{\circ}$. 

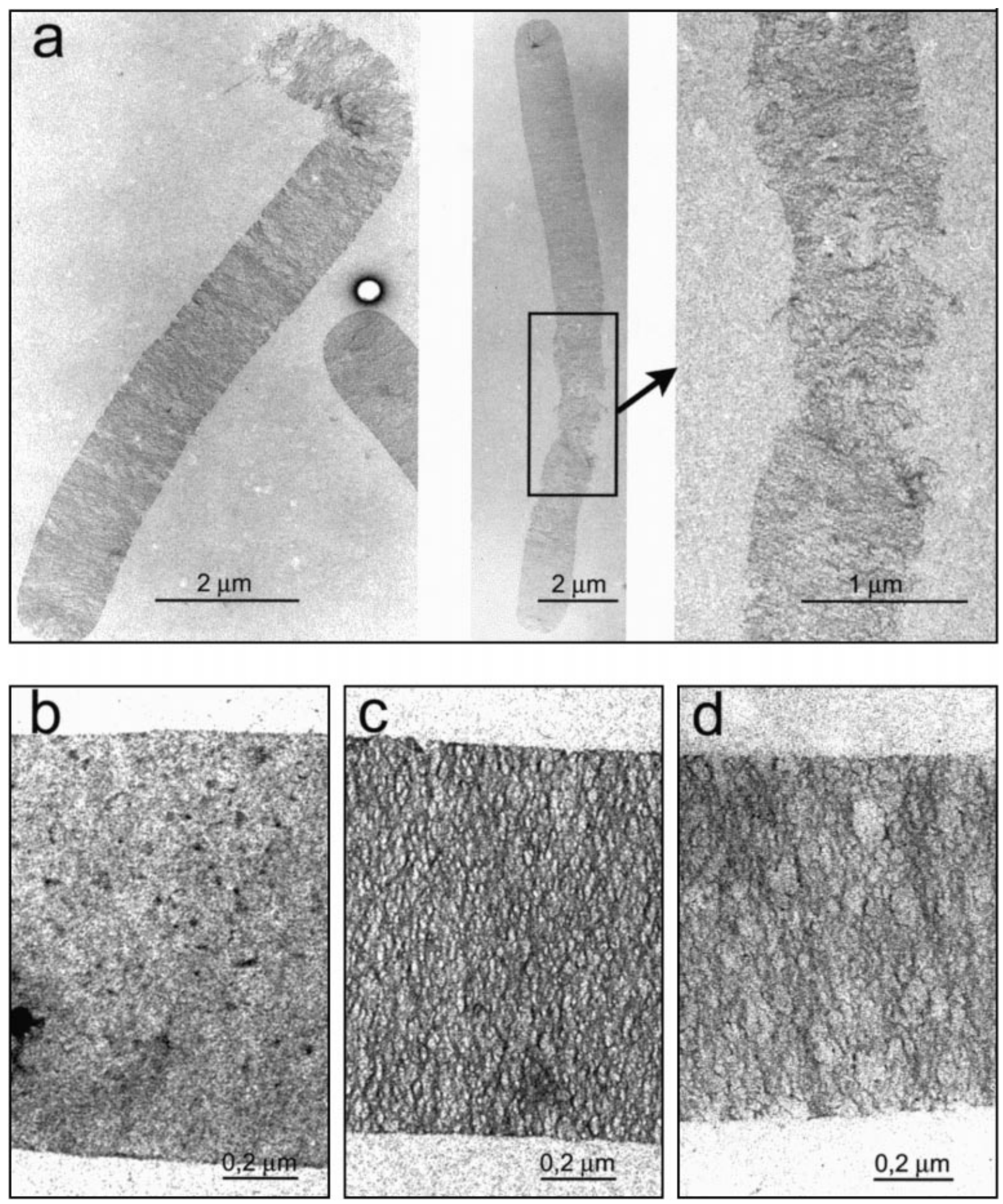

Fig. 3. Electron microscopy of sacculi from cefsulodin-treated filament cells of $E$. coli MC6RP62 (ftsZ) growing at $42{ }^{\circ} \mathrm{C}$. Samples from control and cefsulodin-treated cultures of MC6RP62 growing at $42{ }^{\circ} \mathrm{C}$ were collected as indicated in Fig. 1 and processed for electron microscopy. (a) Cefsulodin-treated sacculi displaying extended damaged areas; (b) control sacculi; (c) cefsulodin-treated sacculi; (d) muramidase-digested control sacculi. 


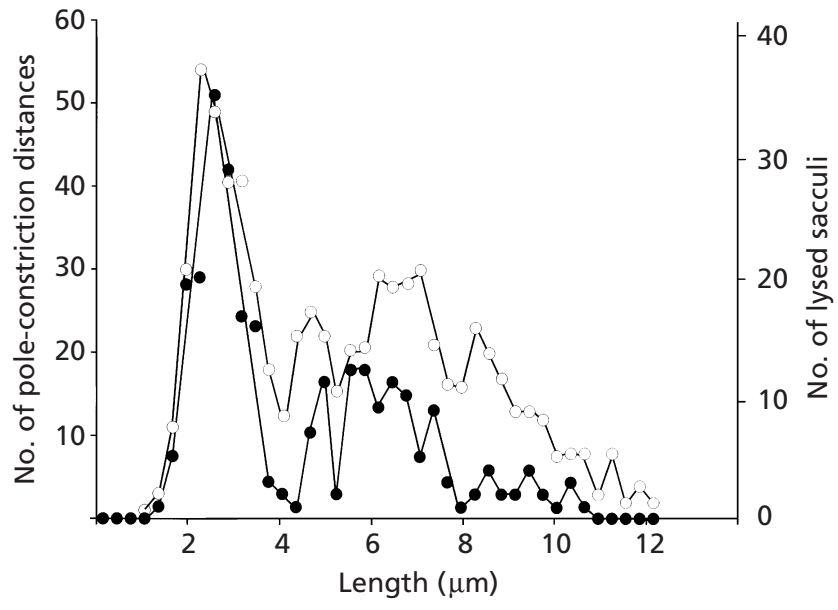

Fig. 4. Comparison of the length distribution of lysed sacculi from a cefsulodin-treated culture of E. coli MC6RP41 ( $f t s l)$ and the distribution of polar distances for constrictions in sacculi from the corresponding control culture. E. coli MC6RP41 was grown as described in Fig. 1 and samples for sacculi purification were collected $60 \mathrm{~min}$ after addition of cefsulodin. Sacculi were prepared for electron microscopy. The lengths of lysed sacculi in the cefsulodin-treated sample were calculated from the rectangular coordinates of both ends as measured with the help of the electron microscope grid positioning device. Polar distances of constrictions in control sacculi were calculated as described in Results, but measuring the distances from each constriction to each cell pole. Calculated values were distributed in $0.3 \mu \mathrm{m}$ size classes. $\bigcirc$, Polar distances of constrictions in control sacculi, 140 sacculi measured; $\bullet$, length of cefsulodin-lysed sacculi, 260 sacculi measured.

\section{ACKNOWLEDGEMENTS}

This work was supported by grant PM97-0148 from the PGC, an institutional grant from Fundación Ramón Areces and a grant from the MPG-CSIC bilateral agreement to M.A.P.

\section{REFERENCES}

Ayala, J. A., Garrido, T., de Pedro, M. A. \& Vicente, M. (1994). Molecular biology of bacterial septation. In Bacterial Cell Wall, pp. 73-101. Edited by J. M. Ghuysen \& R. Hakenbeck. Amsterdam: Elsevier.

Blackman, S. A., Smith, T. J. \& Foster, S. J. (1998). The role of autolysins during vegetative growth of Bacillus subtilis 168. Microbiology 144, 73-82.

Garcia del Portillo, F. \& de Pedro, M. A. (1990). Differential effect of mutational impairment of penicillin-binding proteins $1 \mathrm{~A}$ and 1B on Escherichia coli strains harboring thermosensitive mutations in the cell division genes $f t s A, f t s Q, f t s Z$, and $p b p B . J$ Bacteriol 172, 5863-5870.

Garcia del Portillo, F., de Pedro, M. A., Joseleau-Petit, D. \& D'Ari, R. (1989). Lytic response of Escherichia coli cells to inhibitors of penicillin-binding proteins $1 \mathrm{a}$ and $1 \mathrm{~b}$ as a timed event related to cell division. J Bacteriol 171, 4217-4221.

Glauner, B. \& Höltje, J.-V. (1990). Growth pattern of the murein sacculus of Escherichia coli. J Biol Chem 265, 18988-18996.

Heidrich, C., Templin, M. F., Ursinus, A., Merdanovic, M., Berger, J., Schwarz, H., de Pedro, M. A. \& Höltje, J. -V. (2001). Involvement of $\mathrm{N}$-acetylmuramyl-L-alanine amidases in cell separation and antibiotic-induced autolysis of Escherichia coli. Mol Microbiol 41, 167-178.

Höltje, J.-V. (1995). From growth to autolysis: the murein hydrolases in Escherichia coli. Arch Microbiol 164, 243-254.

Höltje, J.-V. (1996). Molecular interplay of murein synthases and murein hydrolases in Escherichia coli. Microb Drug Resist 2, 99-103.

Höltje, J.-V. (1998). Growth of the stress-bearing and shapemaintaining murein sacculus of Escherichia coli. Microbiol Mol Biol Rev 62, 181-203.

Höltje, J.-V. \& Glauner, B. (1990). Structure and metabolism of the murein sacculus. Res Microbiol 141, 75-89.

Höltje, J.-V. \& Heidrich, C. (2001). Enzymology of elongation and constriction of the murein sacculus of Escherichia coli. Biochimie 83, 103-108.

Ishiguro, E. E. \& Kusser, W. (1988). Regulation of peptidoglycan biosynthesis and antibiotic-induced autolysis in nongrowing Escherichia coli: a preliminary model. In Antibiotic Inhibition of Bacterial Cell Surface Assembly and Function, pp. 189-194. Edited by P. Actor, L. Daneo-Moore, M. L. Higgins, M. R. J. Salton \& G. D. Shockman. Washington, DC: American Society for Microbiology.

Jacoby, G. H. \& Young, K. D. (1991). Cell cycle-independent lysis of Escherichia coli by cefsulodin, an inhibitor of penicillinbinding proteins $1 \mathrm{a}$ and $1 \mathrm{~b}$. J Bacteriol 173, 1-5.

Koch, A. L. (2000). The exoskeleton of bacterial cells (the sacculus): still a highly attractive target for antibacterial agents that will last for a long time. Crit Rev Microbiol 26, 1-35.

Kusser, W. \& Ishiguro, E. E. (1986). Lysis of nongrowing Escherichia coli by combinations of beta-lactam antibiotics and inhibitors of ribosome function. Antimicrob Agents Chemother 29, $451-455$.

Kusser, W. \& Ishiguro, E. E. (1987). Suppression of mutations conferring penicillin tolerance by interference with the stringent control mechanism of Escherichia coli. J Bacteriol 169, 4396-4398.

Leduc, M., Kasra, R. \& van Heijenoort, J. (1982). Induction and control of the autolytic system of Escherichia coli. J Bacteriol 152, 26-34.

Leduc, M. \& van Heijenoort, J. (1980). Autolysis of Escherichia coli. J Bacteriol 142, 52-59.

Lennox, E. S. (1955). Transduction of linked genetic characters of the host by bacteriophage P1. Virology 1, 190-206.

Nanninga, N. (1991). Cell division and peptidoglycan assembly in Escherichia coli. Mol Microbiol 5, 791-795.

Nanninga, N. (1998). Morphogenesis of Escherichia coli. Microbiol Mol Biol Rev 62, 110-129.

de Pedro, M. A., Quintela, J. C., Höltje, J.-V. \& Schwarz, H. (1997). Murein segregation in Escherichia coli. J Bacteriol 179, 28232834.

Powell, J. K. \& Young, K. D. (1991). Lysis of Escherichia coli by beta-lactams which bind penicillin-binding proteins $1 \mathrm{a}$ and $1 \mathrm{~b}$ : inhibition by heat shock proteins. J Bacteriol 173, 4021-4026.

Prats, R. \& de Pedro, M. A. (1989). Normal growth and division of Escherichia coli with a reduced amount of murein. J Bacteriol 171, 3740-3745.

Rogers, H. J., Perkins, H. R. \& Ward, J. B. (1980). Antibiotics affecting bacterial wall synthesis. In Microbial Cell Walls and Membranes, pp. 298-382. Edited by H. J. Rogers, H. R. Perkins \& J. B. Ward. London: Chapman and Hall.

Schwarz, U., Asmus, A. \& Frank, H. (1969). Autolytic enzymes and cell division of Escherichia coli. J Mol Biol 41, 419-429. 
Shockman, G. D. \& Höltje, J.-V. (1994). Microbial peptidoglycan (murein) hydrolases. In Bacterial Cell Wall, pp. 131-166. Edited by J. M. Ghuysen \& R. Hakenbeck. Amsterdam: Elsevier.

Staugaard, P., van den Berg, F. M., Woldringh, C. L. \& Nanninga, N. (1976). Localization of ampicillin-sensitive sites in Escherichia coli by electron microscopy. J Bacteriol 127, 1376-1381.

Tomasz, A. (1974). The role of autolysins in cell death. In Mode of Action of Antibiotics on Microbial Walls and Membranes, pp. 439-447. Edited by M. R. J. Salton \& A. Tomasz. New York: The New York Academy of Sciences.

Tomasz, A. (1983). Murein hydrolases - Enzymes in search of a physiological function? In The Target of Penicillin, pp. 155-163. Edited by R. Hakenbeck, J.-V. Höltje \& H. Labischinski. Berlin : Walter der Gruyter.

Tomasz, A. (1984). Building and breaking of bonds in the cell wall of bacteria - the role for autolysins. In Microbial Cell Wall Synthesis and Autolysis, pp. 3-12. Edited by C. Nombela. Amsterdam: Elsevier.

Tuomanen, E. \& Tomasz, A. (1990). Mechanism of phenotypic tolerance of nongrowing pneumococci to beta-lactam antibiotics. Scand J Infect Dis Suppl 74, 102-112.

Weidel, W. \& Pelzer, H. (1964). Bagshaped macromolecules - a new outlook on bacterial cell walls. Adv Enzymol 26, 193-232.

Wientjes, F. B. \& Nanninga, N. (1991). On the role of the high molecular weight penicillin-binding proteins in the cell cycle of Escherichia coli. Res Microbiol 142, 333-344.

Received 24 May 2001; revised 20 August 2001; accepted 15 September 2001. 